\title{
CONTINUOUS ELECTRON-ENERGY VARIATION OF THE EINDHOVEN RACETRACK MICROTRON
}

\author{
W.H.C. Theuws, J.I.M. Botman, H.L. Hagedoorn \\ Eindhoven University of Technology, Cyclotron Laboratory, \\ P.O. Box 513, 5600MB Eindhoven, The Netherlands.
}

\section{Abstract}

For the Eindhoven racetrack microtron, which has been designed as a fixed-energy accelerator at $75 \mathrm{MeV}$, continuous electron-energy variation between 45 and $90 \mathrm{MeV}$ can be obtained by taking the microtron's orbit pattern as constant and varying certain parameters. The microtron injector is a linac producing electrons between 6 and $12 \mathrm{MeV}$. The microtron cavity potential and the magnetic guide fields must be adapted to the injection energy in order to fulfil the synchronism condition. The racetrack microtron's transversal and longitudinal acceptance are effected by electronvelocity deviations from the speed of light, which are different for each extraction energy. An account of these effects is presented together with the energy-setting measurements by using one of the microtron magnets as a spectrometer.

\section{ISOCHRONISM}

The Eindhoven racetrack microtron, see Fig. 1, has a $10 \mathrm{MeV}, 2998 \mathrm{MHz}$ travelling-wave linac (type M.E.L. SL75/10) as injector. In the microtron a $5 \mathrm{MeV}$ standingwave cavity accelerates the electrons 13 times, such that the extraction energy is $75 \mathrm{MeV}[1]$. Although the microtron has been designed as a fixed-energy machine[2], the extraction energy can be varied continuously without changes in hardware.

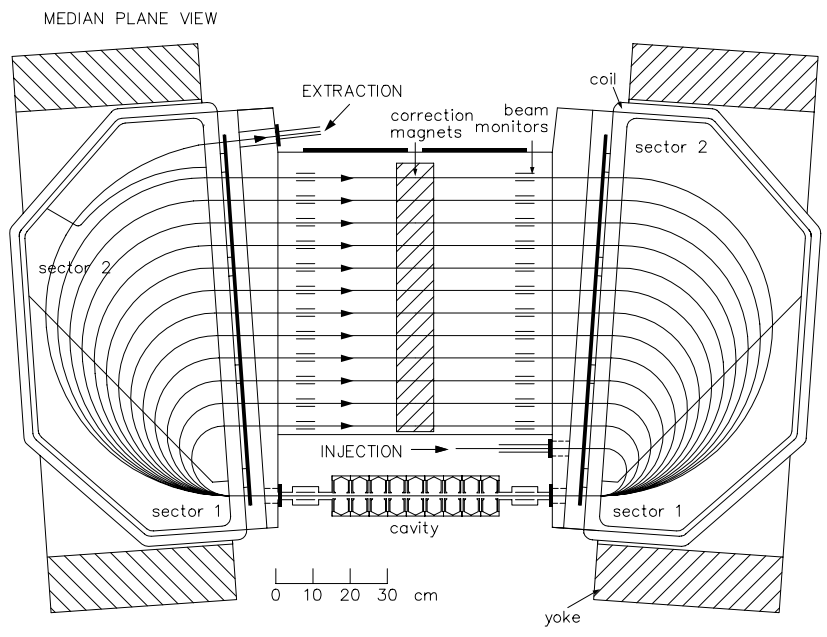

Figure 1: The 10-75 MeV Eindhoven racetrack microtron.

For isochronous acceleration the revolution time of each orbit in the accelerator must be an integer multiple of the
RF-period, $1 / f_{0}$. The isochronism condition for the first orbit is given by:

$$
t_{1}=\frac{2 L}{\beta_{1} c}+\frac{2 \pi}{B_{r} e c^{2}}\left(E_{0}+E_{i n j}+E_{c a v}\right)=\frac{\mu}{f_{0}},
$$

with $L$ is the microtron drift length, $B_{r}$ the resonant magnetic field of the 180 degrees bending magnets, $E_{0}$ the electron's rest energy, $E_{i n j}$ the kinetic energy at injection, $E_{\text {cav }}$ the cavity potential, $\beta_{1}$ the relative velocity in the first orbit with respect to the speed of light, and $\mu$ the initial harmonic number. For isochronism the time-difference between two successive orbits, $n$ and $n-1$, must be an integer multiple of the RF-period as well:

$$
\Delta t=\frac{2 L\left(\beta_{n-1}-\beta_{n}\right)}{\beta_{n-1} \beta_{n} c}+\frac{2 \pi}{B_{r} e c^{2}} E_{c a v}=\frac{\nu}{f_{0}},
$$

where $\nu$ is the incremental harmonic number. This condition can only be fulfilled for all orbits if the electrons are highly relativistic, i.e., $\beta_{n-1}=\beta_{n}=1$. With this assumption two basic relations for a racetrack microtron can be derived, viz.

$$
E_{c a v}=\left(\frac{\nu}{\mu-\nu-2 L f_{0} / c}\right)\left(E_{0}+E_{i n j}\right),
$$

and

$$
B_{r}=\frac{2 \pi f_{0}}{e c^{2}} \frac{E_{c a v}}{\nu}
$$

The frequency, $f_{0}$, and the injection energy, $E_{i n j}$, are dictated by the linac-parameters, and have to be $2998 \mathrm{MHz}$ and $10 \mathrm{MeV}$, respectively. The incremental harmonic number, $\nu$, is chosen 2 in order to get well-separated orbits in the microtron (about $60 \mathrm{~mm}[2]$ ). The cavity potential, $E_{c a v}$, is chosen $5 \mathrm{MeV}$. The drift length, $L$, and consequently the initial harmonic number, $\mu$, have to be sufficiently large to create enough space for the cavity. For $\mu=26$ the drift length, $L$, is given by Eq. (3), which yields $0.99 \mathrm{~m}$. The resonant magnetic field, $B_{r}$, is given by Eq. (4), and yields $0.524 \mathrm{~T}$.

The isochronism condition is slightly violated as the particle velocity is not exactly equal to the speed of light. This implies that it takes a particle a little longer to complete an orbit. For the first orbit the extra time needed is $4.7 \mathrm{ps}$, which corresponds to 5.1 degrees of the RFperiod. For the next orbits this phase-difference becomes less as the particle velocity increases, viz. 3.1, 2.2, 1.6 degrees, etcetera. Isochronism is not effected seriously by these phase-differences, as the stable phase-interval, given by[3]:

$$
\Delta \phi=\arctan \left(\frac{2}{\pi \nu}\right)
$$


equals 18 degrees for the Eindhoven microtron.

The use of two-sector bending magnets, see Fig. 2, instead of homogeneous bending magnets does not violate the isochronism conditions at all. For homogeneous bend-

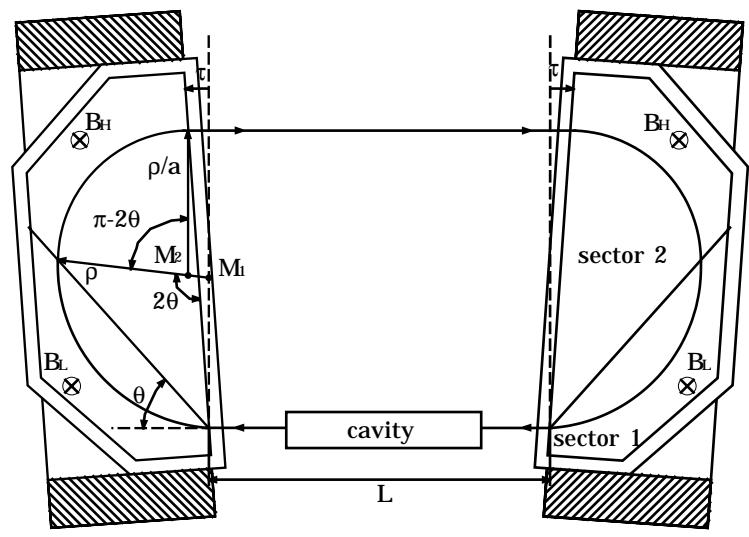

Figure 2: Two-sector bending magnets. The high magnetic field, $B_{H}$, is equal to $a$ times the low magnetic field, $B_{L}$, which is linear with $B_{r}[2]$.

ing magnets the orbit length of the $n^{\text {th }}$ orbit is: $s_{n}=$ $2 L+2 \pi \frac{p_{n}}{B_{r} e}\left(p_{n}\right.$ is the momentum of the $n^{t h}$ orbit). For the two-sector magnet the orbit length is:

$$
s_{n}=2 L+2 \frac{2 \Theta a+\pi-2 \Theta+(a-1) \sin 2 \Theta}{1+\frac{1}{\pi}(a-1)(2 \Theta+\sin 2 \Theta)} \frac{p_{n}}{B_{r} e},
$$

which is linear with momentum just as for homogeneous bending magnets. This implies that the two-sector magnets do not influence isochronism; there is only a different relation between the resonant magnetic field and momentum.

\section{ENERGY VARIATION}

Different combinations of $E_{i n j}, E_{c a v}$ and $B_{r}$ can fulfil the basic microtron Eq. (3) and (4) as well. These three parameters can be changed without changes in hardware. The injection energy is provided by the linac, which can be varied continuously between 6 and $12 \mathrm{MeV}$. The cavity potential and resonant magnetic field can be adapted to the injection energy. The other parameters apparent in equations (3) and (4) can be kept constant.

The microtron's extraction energy is given by

$$
E_{\text {extract }}=E_{i n j}+13 E_{\text {cav }},
$$

in which $E_{c a v} \approx \frac{1}{2} E_{i n j}$ according to Eq. (3). Thus the extraction energy can be varied continuously between about 45 and $90 \mathrm{MeV}$. But, for lower extraction energies isochronism is violated more severely. An account of this effect is presented in the next sections.

\section{TRANSVERSAL ACCEPTANCE}

Energy variation as described in the previous section uses a constant orbit pattern. This means that all 'obstacles' that limit the transversal acceptance are at the same positions relative to the beam. Consequently the transversal acceptance is not influenced by energy variation.

However, this is not exactly true as the orbit pattern does not remain exactly constant. The bending radius, $\rho$, is linear with $\gamma \beta$. So for high energies $\rho$ is linear with $\gamma$, which is linear with energy. But, for lower energies $\beta$ plays a role as $\beta$ is not linear with energy.

Consider the worst-case situation: $E_{i n j}=6 \mathrm{MeV}$, and consequently $E_{\text {cav }} \approx 3 \mathrm{MeV}$. This means that the energy in the first orbit is $9 \mathrm{MeV}$, which means $\beta=0.999$. The relative influence for the bending radius $\rho$ is $1-\beta$, which is $0.1 \%$. As $\rho \approx 0.1 \mathrm{~m}$ for the first orbit, this means that $\delta \rho \approx 0.1 \mathrm{~mm}$. This is negligible with respect to the $10 \mathrm{~mm}$ space, which is available for the beam.

Thus the transversal acceptance is not influenced by energy variation.

\section{LONGITUDINAL ACCEPTANCE}

The longitudinal acceptance is calculated using Dôme's equations describing the dynamics of the synchrotron motion[4].

The phase $\phi_{n}$ and the energy $E_{n}$ are defined just after the $n^{\text {th }}$ cavity passage. The deviation of the phase and the energy from the synchronous value are given by $\Delta \phi_{n}=\phi_{n}-\phi_{s}$ and $\Delta E_{n}=E_{n}-E_{s, n}(n=1,2,3, \ldots)$, respectively. The dynamics of the synchrotron motion is described by the finite difference equations:

$$
\Delta \phi_{n+1}=\Delta \phi_{n}+\frac{2 \pi \nu \Delta \epsilon_{n}}{\beta_{n}},
$$

and

$$
\begin{array}{r}
\left(\frac{\Delta \epsilon}{\omega_{s}}\right)_{n+1}=\left(\frac{\Delta \epsilon}{\omega_{s}}\right)_{n}+ \\
+\left(\frac{1}{\omega_{s}}\right)_{n}\left(\frac{\cos \left(\phi_{s}+\Delta \phi_{n+1}\right)-\cos \phi_{s}}{\cos \phi_{s}}\right),
\end{array}
$$

with $\Delta \epsilon_{n}=\Delta E_{n} / E_{\text {cav }}$.

However, perfect isochronism is assumed in these equations. The violation of isochronism as a result of the fact that the electrons do not exactly have the speed of light (see Section 1) can be incorporated by adding a term $\Delta \phi_{\beta, n}$, which accounts for this effect, to the right-hand side of Eq. (8).

The longitudinal acceptance is calculated from Dôme's equations. For a regular grid of combinations of initial deviations $\Delta \phi_{1}$ and $\Delta \epsilon_{1}$ Dôme's equations are applied 13 times. If $\Delta \epsilon_{n}$ remains small during these calculations the position in longitudinal phase-space is considered as accepted. The longitudinal acceptance is linear with the extraction energy from $1.0 \mathrm{deg} \mathrm{MeV}$ for $E_{\text {extract }}=45 \mathrm{MeV}$ 
to $2.1 \mathrm{deg} \mathrm{MeV}$ for $E_{\text {extract }}=90 \mathrm{MeV}$. However, the acceptance plot is not perfectly centred around the origin. It is more off-centre for lower energies. This implies that an initial phase off-set and injection-energy off-set have to be applied. For extremely high energies the longitudinal acceptance stabilizes at about $11 \mathrm{deg} \mathrm{MeV}$, but this is for extraction energies of about $1 \mathrm{GeV}$ or higher, which is practically impossible to achieve with the Eindhoven racetrack microtron.

\section{SPECTROMETER}

When the extraction energy is chosen the injection energy and cavity potential can be calculated from Eqs. (3) and (7). The initial phase off-set and injection-energy off-set of the electron bunches can be determined from longitudinalacceptance calculations (see Section 4). Then the injection energy, the cavity potential and the initial phase off-set are to be adjusted. The injection energy is determined by the injection line; the cavity potential by an RF-attenuator; and the phase by an RF phase-shifter.

The left-hand microtron magnet will be used to measure these three energy parameters[5]. This magnet is excited such that the beam is extracted immediately after the first cavity passage without recirculating in the microtron, see Fig. 3. The excitation current of the magnet in combination

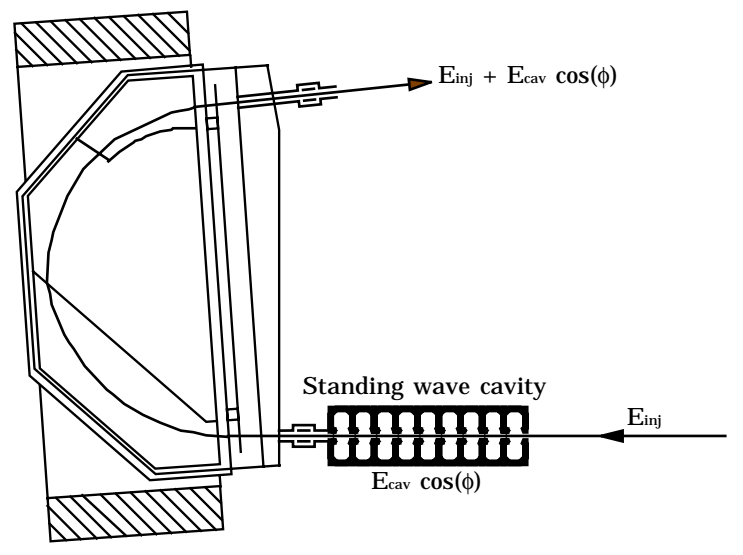

Figure 3: Left-hand RTM-magnet used as spectrometer.

with the beam-position monitors just before and behind the magnet give the mean energy of the beam. This energy is measured for different settings of the phase shifter, which results in a sine-like form of the energy as a function of phase,

$$
E=E_{i n j}+E_{\text {cav }} \cos \phi .
$$

The injection energy, $E_{i n j}$, is the mean of the function. The cavity potential, $E_{\text {cav }}$, is the amplitude of the harmonic part. Moreover, the phase-difference, $\phi$, is calibrated.

The absolute measurement accuracy of the 'spectrometer' is estimated to be $2 \%$. The relative measurement accuracy is only about $0.2 \%$, as the alignment errors of the beam-position monitors just before and behind the magnet and the absolute value of the magnetic field do not influence the relative accuracy.

\section{CONCLUSIONS}

Continuous electron-energy variation is very well possible with the Eindhoven linac-racetrack microtron combination without changes in hardware. The extraction energy can be varied between about 45 and $90 \mathrm{MeV}$. For lower extraction energies the longitudinal acceptance of the racetrack microtron decreases as the isochronism conditions are slightly violated by particle-velocity deviations from the speed of light. Moreover, the extraction energy cannot be varied dynamically as the microtron parameters that have to be varied are not controlled by dynamic control loops.

The injection energy and cavity potential can be measured within about $2 \%$ by using one of the microtron magnets as spectrometer. These measurements are not accurate enough for proper acceleration in the microtron[2], however the exact values are not important as long as $\frac{E_{c a v}}{E_{i n j}}$ is in agreement with Eq. (3). The relative measurement accuracy of the spectrometer is estimated to about $0.2 \%$, which is accurate enough. The resonant magnetic field can be adapted to the exact values according to Eq. (4).

\section{REFERENCES}

[1] Theuws, W.H.C., Botman, J.I.M., Hagedoorn, H.L., Leeuw, R.W. de, Timmermans, C.J., The $75 \mathrm{MeV}$ racetrack microtron Eindhoven, Proc. Lin. Acc. Conf., Geneva (1996) pp. $92-94$.

[2] Webers, G.A., Design of an electron optical-system for a $75 \mathrm{MeV}$ racetrack microtron, Ph.D Thesis, Eindhoven University of Technology (1994).

[3] Kapitza, S.P., Melekhin, V.N., The microtron, translated from Russian by I.N. Sviatoslavsky, Harwoord Academic Publishers, London (1978).

[4] Dôme, G., Theory of RF acceleration, Proc. Cern Accelerator School, advanced accelerator physics (1980) pp. 110-143.

[5] Theuws, W.H.C., Leeuw, R.W. de, Gossens, L.W.A.M., Spoek, P.M., Botman, J.I.M., Timmermans, C.J., Hagedoorn, H.L., Diagnostic system of the Eindhoven linacracetrack microtron combination, Proc. Eur. Part. Acc. Conf., Barcelona (1996) pp. 1615-1617. 